ARTICLE

DOI: $10.1038 / \mathrm{s} 41467-018-04536-7$

OPEN

\title{
Energy-temperature uncertainty relation in quantum thermodynamics
}

\author{
H.J.D. Miller ${ }^{1} \&$ J. Anders ${ }^{1}$
}

It is known that temperature estimates of macroscopic systems in equilibrium are most precise when their energy fluctuations are large. However, for nanoscale systems deviations from standard thermodynamics arise due to their interactions with the environment. Here we include such interactions and, using quantum estimation theory, derive a generalised thermodynamic uncertainty relation valid for classical and quantum systems at all coupling strengths. We show that the non-commutativity between the system's state and its effective energy operator gives rise to quantum fluctuations that increase the temperature uncertainty. Surprisingly, these additional fluctuations are described by the average Wigner-YanaseDyson skew information. We demonstrate that the temperature's signal-to-noise ratio is constrained by the heat capacity plus a dissipative term arising from the non-negligible interactions. These findings shed light on the interplay between classical and non-classical fluctuations in quantum thermodynamics and will inform the design of optimal nanoscale thermometers.

\footnotetext{
${ }^{1}$ Department of Physics and Astronomy, University of Exeter, Stocker Road, Exeter EX4 4QL, UK. Correspondence and requests for materials should be addressed to H.J.D.M. (email: hm419@exeter.ac.uk) or to J.A. (email: janet@qipc.org)
} 
D ohr suggested that there should exist a form of complementarity between temperature and energy in thermodynamics similar to that of position and momentum in quantum theory ${ }^{1}$. His reasoning was that in order to assign a definite temperature $T$ to a system it must be brought in contact with a thermal reservoir, in which case the energy $U$ of the system fluctuates due to exchanges with the reservoir. On the other hand, to assign a sharp energy to the system it must be isolated from the reservoir, rendering the system's temperature $T$ uncertain. Based on this heuristic argument Bohr conjectured the thermodynamic uncertainty relation:

$$
\Delta \beta \geq \frac{1}{\Delta U}
$$

with $\beta=\left(k_{\mathrm{B}} T\right)^{-1}$ the inverse temperature. While Eq. (1) has since been derived in various settings ${ }^{2-9}$, it was Mandelbrot who first based the concept of fluctuating temperature on the theory of statistical inference. Concretely, for a thermal system in canonical equilibrium, $\Delta \beta$ can be interpreted as the standard deviation associated with estimates of the parameter $\beta$. Mandelbrot proved that Eq. (1) sets the ultimate limit on simultaneous estimates of energy and temperature in classical statistical physics ${ }^{2}$.

The notion of fluctuating temperature has proved to be fundamental in the emerging field of quantum thermometry, where advances in nanotechnology now allow temperature sensing at sub-micron scales ${ }^{10-22}$. Using the tools of quantum metrology ${ }^{23}$, the relation Eq. (1) can also be derived for weakly coupled quantum systems ${ }^{11,12,14}$, where the equilibrium state is best described by the canonical ensemble. Within the grand-canonical ensemble the impact of the indistinguishability of quantum particles on the estimation of temperature and the chemical potential has also been explored ${ }^{24}$. Relation Eq. (1) informs us that when designing an accurate quantum thermometer one should search for systems with Hamiltonians that produce a large energy variance $^{14}$.

Recently there has been an emerging interest into the effects of strong coupling on temperature estimation ${ }^{13,15,25}$. At the nanoscale the strength of interactions between the system and the reservoir may become non-negligible, and the local equilibrium state of the system will not be of Gibbs form ${ }^{26,27}$. In this regime thermodynamics needs to be adapted as the equilibrium properties of the system must now depend on the interaction energy $^{28-40}$. We will see that the internal energy $U$ and its fluctuations $\Delta U$ are determined by a modified internal energy operator, denoted by $\hat{E}_{\mathcal{S}}^{*}$, that differs from the bare Hamiltonian of the system ${ }^{35,39}$. This modification brings into question the validity of Eq. (1) for general classical and quantum systems, and the aim of this paper is to investigate the impact of strong coupling on the thermodynamic uncertainty relation.

Taking into account quantum properties of the effective internal energy operator and its temperature dependence, we here derive the general thermodynamic uncertainty principle valid at all coupling strengths. Formally this result follows from a general upper bound on the quantum Fisher information (QFI) for exponential states. We prove that quantum fluctuations arising from coherences between energy states of the system lead to increased fluctuations in the underlying temperature. Most interestingly, the non-classical modifications to Eq. (1) are quantified by the average Wigner-Yanase-Dyson (WYD) skew information $^{41-44}$, which is a quantity closely linked to measures of coherence, asymmetry and quantum speed limits ${ }^{45,46}$. We then demonstrate that the skew information is also linked to the heat capacity of the system through a modified fluctuation-dissipation relation (FDR). This result is used to find an upper bound on the achievable signal-to-noise ratio of an unbiased temperature estimate, and we illustrate our bound with an example of a damped harmonic oscillator.

\section{Results}

The Wigner-Yanase-Dyson skew information. Our analysis throughout the paper will rely on distinguishing between classical and non-classical fluctuations of observables in quantum mechanics, and we first present a framework for quantifying these different forms of statistical uncertainty for arbitrary mixed states.

Let us consider a quantum state $\hat{\rho}$ and an observable $\hat{A}$. Wigner and Yanase considered the problem of quantifying the quantum uncertainty in observable $\hat{A}$ for the case where $\hat{\rho}$ is mixed ${ }^{41}$. However, they observed that the standard measure of uncertainty, namely the variance $\operatorname{Var}[\hat{\rho}, \hat{A}]:=\operatorname{tr}\left[\hat{\rho} \delta \hat{A}^{2}\right]$ with $\delta \hat{A}=\hat{A}-\langle\hat{A}\rangle$, contains classical contributions due to mixing, and thus fails to fully quantify the non-classical fluctuations in the observable $\hat{A}$. This problem can be resolved by finding a quantum measure of uncertainty $Q[\hat{\rho}, \hat{A}]$ and classical measure $K[\hat{\rho}, \hat{A}]$ such that the variance can be partitioned according to

$$
\operatorname{Var}[\hat{\rho}, \hat{A}]=Q[\hat{\rho}, \hat{A}]+K[\hat{\rho}, \hat{A}] .
$$

Following the framework introduced by Luo ${ }^{47}$, these functions are required to fulfil three conditions: (i) both terms should be non-negative, $Q[\hat{\rho}, \hat{A}] \geq 0$ and $K[\hat{\rho}, \hat{A}] \geq 0$, so that they can be interpreted as forms of statistical uncertainty, (ii) if the state $\hat{\rho}$ is pure, then $Q[\hat{\rho}, \hat{A}]=\operatorname{Var}[\hat{\rho}, \hat{A}]$ while $K[\hat{\rho}, \hat{A}]=0$ as all uncertainty should be associated to quantum fluctuations alone, (iii) $Q[\hat{\rho}, \hat{A}]$ must be convex with respect to $\hat{\rho}$, so that it decreases under classical mixing. Correspondingly, $K[\hat{\rho}, \hat{A}]$ must be concave with respect to $\hat{\rho}$.

The following function, known as the WYD skew information $^{41}$ was shown to be a valid measure of quantum uncertainty:

$$
Q_{a}[\hat{\rho}, \hat{A}]:=-\frac{1}{2} \operatorname{tr}\left[\left[\hat{A}, \hat{\rho}^{a}\right]\left[\hat{A}, \hat{\rho}^{1-a}\right]\right] ; \quad a \in(0,1),
$$

with the complementary classical uncertainty given by

$$
K_{a}[\hat{\rho}, \hat{A}]:=\operatorname{tr}\left[\hat{\rho}^{a} \delta \hat{A} \hat{\rho}^{1-a} \delta \hat{A}\right] ; \quad a \in(0,1) .
$$

While conditions (i) and (ii) are easily verified, the convexity/ concavity of $Q_{a}[\hat{\rho}, \hat{A}]$ and $K_{a}[\hat{\rho}, \hat{A}]$ respectively can be proven using Lieb's concavity theorem.

The presence of the parameter $a$ demonstrates that there is no unique way of separating the quantum and classical contributions to the variance. We here follow the suggestion made in refs. ${ }^{43,44}$ and average over the interval $a \in(0,1)$ to define two new quantities:

$$
\begin{aligned}
& Q[\hat{\rho}, \hat{A}]:=\int_{0}^{1} \mathrm{~d} a Q_{a}[\hat{\rho}, \hat{A}], \\
& K[\hat{\rho}, \hat{A}]:=\int_{0}^{1} \mathrm{~d} a K_{a}[\hat{\rho}, \hat{A}] .
\end{aligned}
$$

It is not only the $Q_{a}[\hat{\rho}, \hat{A}]$ and $K_{a}[\hat{\rho}, \hat{A}]$ that separate the quantum and classical fluctuations of a quantum observable $\hat{A}$ in a state $\hat{\rho}$ according to Eq. (2), but also the averaged $Q[\hat{\rho}, \hat{A}]$ and $K[\hat{\rho}, \hat{A}]$. This follows from the linearity of the integrals in Eqs. (5) and (6) which also preserve the conditions (i)-(iii). Throughout the remainder of the paper we will consider $Q[\hat{\rho}, \hat{A}]$ and $K[\hat{\rho}, \hat{A}]$ as the relevant measures of quantum and classical uncertainty, respectively. While this may appear to be an arbitrary choice, we 
will subsequently prove that the average skew information is intimately connected to thermodynamics.

Bound on quantum Fisher information for exponential states. We now prove that the average skew information is linked to the quality of a parameter estimate for a quantum exponential state. A quantum exponential state is of the form $\hat{\rho}_{\theta}=e^{-\hat{A}_{\theta}} / Z_{\theta}$ where $Z_{\theta}=\operatorname{tr}\left[e^{-\hat{A}_{\theta}}\right]$ and $\hat{A}_{\theta}$ is a hermitian operator that is here assumed to depend analytically on a smooth parameter $\theta$. For any state of full rank, an operator $\hat{A}_{\theta}$ can be found such that the state can be expressed in this form, i.e. all full rank states are exponential states.

We first recall the standard setup for estimating the parameter $\theta^{48}$. First one performs a POVM measurement $\hat{M}(\xi)$, where $\int \mathrm{d} \xi \hat{M}(\xi)=\hat{\mathbb{1}}$ and $\xi$ denotes the outcomes of the measurement which may be continuous or discrete. The probability of obtaining a particular outcome is $p(\xi \mid \theta)=\operatorname{tr}\left[\hat{M}(\xi) \hat{\rho}_{\theta}\right]$. The measurement is repeated $n$ times with outcomes $\left\{\xi_{1}, \xi_{2}, . . \xi_{n}\right\}$, and one constructs a function $\tilde{\theta}=\tilde{\theta}\left(\xi_{1}, \xi_{2}, . . \xi_{n}\right)$ that estimates the true value of the parameter. We denote the average estimate by $\langle\tilde{\theta}\rangle$, where $\langle(.)\rangle=.\int \mathrm{d} \xi_{1} \ldots \mathrm{d} \xi_{n} p\left(\xi_{1} \mid \theta\right) \ldots p\left(\xi_{n} \mid \theta\right)(.$.$) , and assume the$ estimate is unbiased, i.e. $\langle\tilde{\theta}\rangle=\theta$. In this case the mean-squared error in the estimate is equivalent to the variance, which is denoted by $\Delta \theta^{2}=\left\langle\tilde{\theta}^{2}\right\rangle-\theta^{2}$.

The celebrated quantum Cramér-Rao inequality sets a lower bound on $\Delta \theta$, optimised over all possible POVMs and estimator functions $^{23,48-50}$ :

$$
\Delta \theta \geq \frac{1}{\sqrt{n F(\theta)}},
$$

where $F(\theta)$ is the QFI. The bound becomes tight in the asymptotic limit $n \rightarrow \infty^{23}$. If the exponential state belongs to the so-called 'exponential family', which is true if $\hat{A}_{\theta}=\theta \hat{X}+\hat{Y}$ for commuting operators $\hat{X}, \hat{Y}$, then the bound is also tight in the single-shot limit $(n=1)^{50}$. The QFI with respect to $\theta$ is defined by $F(\theta):=\operatorname{tr}\left[\hat{\rho}_{\theta} \hat{L}_{\theta}^{2}\right]$, where $\hat{L}_{\theta}$ is the symmetric logarithmic derivative which uniquely satisfies the operator equation $\partial_{\theta} \hat{\rho}_{\theta}=\frac{1}{2}\left\{\hat{L}_{\theta}, \hat{\rho}_{\theta}\right\}^{49}$. Here $\{. . . .$.$\} denotes the anti-commutator.$

We now state a general upper bound on $F(\theta)$ valid for any exponential state:

Theorem 1: For an exponential state $\hat{\rho}_{\theta}=e^{-\hat{A}_{\theta}} / Z_{\theta}$ the QFI with respect to the parameter $\theta$ is bounded by

$$
F(\theta) \leq K\left[\hat{\rho}_{\theta}, \hat{B}_{\theta}\right]
$$

Here $K\left[\hat{\rho}_{\theta}, \hat{B}_{\theta}\right]$ is defined in Eq. (6), and $\hat{B}_{\theta}$ is the hermitian observable $\hat{B}_{\theta}:=\partial_{\theta} \hat{A}_{\theta}$. The bound becomes tight in the limits where $\hat{\rho}_{\theta}$ is maximally mixed.

This theorem demonstrates that the strictly classical fluctuations in $\hat{B}_{\theta}$ constrain the achievable precision in estimates of $\theta$. The proof of the theorem is outlined in the 'Methods' section.

We note that for states $\hat{\sigma}_{\theta}$ that fulfil the von-Neumann equation $\partial_{\theta} \hat{\sigma}_{\theta}=-i\left[\hat{A}_{\theta}, \hat{\sigma}_{\theta}\right]$ a connection between skew information $Q_{1 / 2}\left[\hat{\sigma}_{\theta}, \hat{A}_{\theta}\right]$ and parameter estimation has previously been made by Luo ${ }^{51}$. While the particular dependence on $\theta$ implied by this equation is relevant for unitary parameter estimation ${ }^{23}$, this dependence will not be relevant for temperature estimation since thermal states do not generally fulfil this von-Neumann equation. In contrast, we will see in the next section that Theorem 1 has implications for the achievable precision in determining temperature.
Generalised thermodynamic uncertainty relation. We will now use the results of the previous section to derive an uncertainty relation between energy and temperature for a quantum system strongly interacting with a reservoir. To achieve this we will first discuss the appropriate energy operator for such a system, and then proceed to generalise Eq. (1).

A quantum system $\mathcal{S}$ that interacts with a reservoir $\mathcal{R}$ is described by a Hamiltonian

$$
\hat{H}_{\mathcal{S} \cup \mathcal{R}}:=\hat{H}_{\mathcal{S}} \otimes \hat{\mathbb{I}}_{\mathcal{R}}+\hat{\mathbb{I}}_{\mathcal{S}} \otimes \hat{H}_{\mathcal{R}}+\hat{V}_{\mathcal{S} \cup \mathcal{R}}
$$

where $\hat{H}_{\mathcal{S}}$ and $\hat{H}_{\mathcal{R}}$ are the bare Hamiltonians of $\mathcal{S}$ and $\mathcal{R}$ respectively, while $\hat{V}_{\mathcal{S} \cup \mathcal{R}}$ is an interaction term of arbitrary strength. We will consider situations where the environment is large compared to the system, i.e. the operator norms fulfil $\left\|\hat{H}_{\mathcal{R}}\right\| \gg\left\|\hat{H}_{\mathcal{S}}\right\|,\left\|\hat{V}_{\mathcal{S} \cup \mathcal{R}}\right\|$. We make no further assumptions about the relative size of the coupling $\left\|\hat{V}_{\mathcal{S} \cup \mathcal{R}}\right\|$ between the system and the environment, and the system's bare energy $\left\|\hat{H}_{\mathcal{S}}\right\|$. The global equilibrium state at temperature $T$ for the total Hamiltonian $\mathcal{S} \cup \mathcal{R}$ is of Gibbs form $\hat{\pi}_{\mathcal{S} \cup \mathcal{R}}(T)=e^{-\beta \hat{H}_{\mathcal{S} \cup \mathcal{R}}} / \mathcal{Z}_{\mathcal{S} \cup \mathcal{R}}$ where $\beta=\left(k_{\mathrm{B}} T\right)^{-1}$ and $\mathcal{Z}_{\mathcal{S} \cup \mathcal{R}}=\operatorname{tr}_{\mathcal{S} \cup \mathcal{R}}\left[e^{-\beta \hat{H}_{\mathcal{S} \cup \mathcal{R}}}\right]$ is the partition function for $\mathcal{S} \cup \mathcal{R}$. The Boltzmann constant $k_{\mathrm{B}}$ will be set to unity throughout.

Due to the presence of the interaction term the reduced state of $\mathcal{S}$, denoted $\hat{\pi}_{\mathcal{S}}(T)=\operatorname{tr}_{\mathcal{R}}\left[\hat{\pi}_{\mathcal{S} \cup \mathcal{R}}(T)\right]$, is generally not thermal with respect to $\hat{H}_{\mathcal{S}}$, unless the coupling is sufficiently weak, i.e. $\left\|\hat{H}_{\mathcal{S}}\right\| \gg\left\|\hat{V}_{\mathcal{S} \cup \mathcal{R}}\right\|$. Therefore the partition function determined by $\hat{H}_{\mathcal{S}}$ can no longer be used to calculate the internal energy of the system ${ }^{35}$. To resolve this issue one can rewrite the state of $\mathcal{S}$ as an effective Gibbs state $\hat{\pi}_{\mathcal{S}}(T):=e^{-\beta \hat{H}_{\mathcal{S}}^{*}(T)} / \mathcal{Z}_{\mathcal{S}}^{*}$, where

$$
\hat{H}_{\mathcal{S}}^{*}(T):=-\frac{1}{\beta} \ln \left(\frac{\operatorname{tr}_{\mathcal{R}}\left[e^{-\beta \hat{H}_{\mathcal{S} \cup \mathcal{R}}}\right]}{\operatorname{tr}_{\mathcal{R}}\left[e^{-\beta \hat{H}_{\mathcal{R}}}\right]}\right),
$$

is the Hamiltonian of mean force $28,30-37,39,40$. The operator $\hat{H}_{\mathcal{S}}^{*}(T)$ acts as a temperature-dependent effective Hamiltonian describing the equilibrium properties of $\mathcal{S}$ through the effective partition function $\mathcal{Z}_{\mathcal{S}}^{*}=\operatorname{tr}_{\mathcal{S}}\left[e^{-\beta \hat{H}_{\mathcal{S}}^{*}(T)}\right]$. The free energy associated with $\mathcal{Z}_{\mathcal{S}}^{*}$ also appears in the open system fluctuation relations ${ }^{30,52}$

The internal energy of $\mathcal{S}$ can be computed from this partition function via $U_{\mathcal{S}}(T):=-\partial_{\beta} \ln \mathcal{Z}_{\mathcal{S}}^{*}$. It is straightforward to show that $U_{\mathcal{S}}(T)$ is just the difference between the total energy, $U_{\mathcal{S} \cup \mathcal{R}}=-\partial_{\beta} \ln \mathcal{Z}_{\mathcal{S} \cup \mathcal{R}}$, and the energy of the reservoir, $\tilde{U}_{\mathcal{R}}=$ $-\partial_{\beta} \ln \mathcal{Z}_{\mathcal{R}}$ with $\mathcal{Z}_{\mathcal{R}}=\operatorname{tr}_{\mathcal{R}}\left[e^{-\beta \hat{H}_{\mathcal{R}}}\right]$, in the absence of any coupling to $\mathcal{S}$, i.e. $U_{\mathcal{S}}(T)=U_{\mathcal{S} \cup \mathcal{R}}(T)-\tilde{U}_{\mathcal{R}}(T)$. In other words, $U_{\mathcal{S}}(T)$ is the energy change induced from immersing the subsystem $\mathcal{S}$ into the composite state $\mathcal{S} \cup \mathcal{R}^{29,36}$.

Seifert has remarked ${ }^{35}$ that $U_{\mathcal{S}}(T)$ can be expressed as an expectation value, $U_{\mathcal{S}}(T)=\left\langle\hat{E}_{\mathcal{S}}^{*}(T)\right\rangle$, of the following observable:

$$
\hat{E}_{\mathcal{S}}^{*}(T):=\partial_{\beta}\left[\beta \hat{H}_{\mathcal{S}}^{*}(T)\right] \text {. }
$$

One can interpret $\hat{E}_{\mathcal{S}}^{*}(T)$ as the effective energy operator describing the system, and we will refer to its eigenstates as "the system energy states". The introduction of this operator allows one to consider fluctuations in the energy $\Delta U_{\mathcal{S}}=\sqrt{\operatorname{Var}\left[\hat{\pi}_{\mathcal{S}}, \hat{E}_{\mathcal{S}}^{*}\right]}$ It is important to note that $\hat{E}_{\mathcal{S}}^{*}(T)$ depends explicitly on the coupling $\hat{V}_{\mathcal{S} \cup \mathcal{R}}$ and the temperature $T$. 
Our first observation is that, in general, $\hat{E}_{S}^{*}(T)$ differs from both the bare system Hamiltonian $\hat{H}_{\mathcal{S}}$ and the mean force Hamiltonian $\hat{H}_{\mathcal{S}}^{*}(T)$. Indeed, this effective energy operator for the system contains the bare energy part as well as an energetic contribution from the coupling, $\hat{E}_{\mathcal{S}}^{*}(T)=$ $\hat{H}_{\mathcal{S}}+\partial_{\beta}\left[\beta\left(\hat{H}_{\mathcal{S}}^{*}(T)-\hat{H}_{\mathcal{S}}\right)\right]$. Moreover, $\hat{E}_{\mathcal{S}}^{*}(T)$ does not even commute with $\hat{H}_{\mathcal{S}}$ and $\hat{H}_{\mathcal{S}}^{*}(T)$. This non-commutativity implies that the state $\hat{\pi}_{\mathcal{S}}(T)$ exists in a superposition of energy states, aside from the trivial situation in which $\left[\hat{H}_{\mathcal{S}}+\hat{H}_{\mathcal{R}}, \hat{V}_{\mathcal{S} \cup \mathcal{R}}\right]=0$. As expected, in the limit of weak coupling $\hat{E}_{\mathcal{S}}^{*}(T)$ reduces to the bare Hamiltonian $\hat{H}_{\mathcal{S}}$.

We are now ready to state the generalised thermodynamic uncertainty relations for strongly coupled quantum systems. Following the approach taken by De Pasquale et al. ${ }^{13}$, we consider the QFI $F_{\mathcal{S}}(\beta)$ associated with the inverse temperature $\beta$. According to the quantum Cramér-Rao bound this functional quantifies the minimum extent to which the inverse temperature fluctuates from the perspective of $\mathcal{S}$, and we denote these fluctuations by $\Delta \beta_{\mathcal{S}}$. Given that the state of $\mathcal{S}$ takes the form $\hat{\pi}_{\mathcal{S}}(T):=e^{-\beta \hat{H}_{\mathcal{S}}^{*}(T)} / \mathcal{Z}_{\mathcal{S}}^{*}$ we can immediately apply Theorem 1 by identifying $\hat{B}_{\theta}=\hat{E}_{\mathcal{S}}^{*}(T)$ with $\theta=\beta$, leading to $F_{\mathcal{S}}(\beta) \leq K\left[\hat{\pi}_{\mathcal{S}}, \hat{E}_{\mathcal{S}}^{*}\right]$. Applying Eq. (7) for the single-shot case $(n=1)$ and using the fact that $K\left[\hat{\pi}_{\mathcal{S}}, \hat{E}_{\mathcal{S}}^{*}\right]=\Delta U_{\mathcal{S}}^{2}-Q\left[\hat{\pi}_{\mathcal{S}}, \hat{E}_{\mathcal{S}}^{*}\right]$, we obtain the following thermodynamic uncertainty relation:

$$
\Delta \beta_{\mathcal{S}} \geq \frac{1}{\sqrt{\Delta U_{\mathcal{S}}^{2}-Q\left[\hat{\pi}_{\mathcal{S}}, \hat{E}_{\mathcal{S}}^{*}\right]}} \geq \frac{1}{\Delta U_{\mathcal{S}}} .
$$

This is the main result of the paper and represents the strongcoupling generalisation of Eq. (1). It can be seen that the bound on the uncertainty in the inverse temperature is increased whenever quantum energy fluctuations are present. These additional fluctuations are quantified by the non-negative $Q\left[\hat{\pi}_{\mathcal{S}}, \hat{E}_{\mathcal{S}}^{*}\right]$, increasing which implies a larger lower bound on $\Delta \hat{\beta}_{\mathcal{S}}$. One recovers the usual uncertainty relation when $Q\left[\hat{\pi}_{\mathcal{S}}, \hat{E}_{\mathcal{S}}^{*}\right]$ can be neglected, which is the case when the interaction commutes with the bare Hamiltonians of $\mathcal{S}$ and $\mathcal{R}$ or when the interaction is sufficiently weak. We note that $Q\left[\hat{\pi}_{\mathcal{S}}, \hat{E}_{\mathcal{S}}^{*}\right]$ vanishes for classical systems and Eq. (12) reduces to the original uncertainty relation Eq. (1), but with energy fluctuations quantified by $\hat{E}_{\mathcal{S}}^{*}$ instead of the bare Hamiltonian $\hat{H}_{\mathcal{S}}$.

If one repeats the experiment $n$ times, then the uncertainty in the estimate can be improved by a factor of $1 / \sqrt{n}^{48}$. We remark that in the weak coupling limit, where $\hat{H}_{\mathcal{S}}^{*}(T) \simeq \hat{H}_{\mathcal{S}}$, the state of $\mathcal{S}$ belongs to the exponential family, and hence the bound on $\Delta \beta_{\mathcal{S}}$ becomes tight for a single measurement in agreement with Eq. (1). However, when $\hat{V}_{\mathcal{S} \cup \mathcal{R}}$ is non-negligible the Hamiltonian of mean force cannot generally be expressed in the linear form $\beta \hat{H}_{\mathcal{S}}^{*}(T)=\beta \hat{X}_{\mathcal{S}}+\hat{Y}_{\mathcal{S}}$. This means in general it is necessary to take the asymptotic limit in order to saturate Eq. (12).

Fluctuation-Dissipation relation beyond weak-coupling. We now detail the impact of strong interactions on the heat capacity of the quantum system and the implications for the precision of temperature measurements. For a fixed volume of the system, the heat capacity is defined as the temperature derivative of the internal energy $U_{\mathcal{S}}(T)^{31,32}$, i.e.

$$
C_{\mathcal{S}}(T):=\frac{\partial U_{\mathcal{S}}}{\partial T}
$$

In standard thermodynamics where the system is described by a Gibbs state the FDR states that the heat capacity is proportional to the fluctuations in energy, i.e. $C_{\mathcal{S}}(T)=\Delta U_{\mathcal{S}}^{2} / T^{2}$. However, example studies of open quantum systems of the form Eq. (9) have shown that the heat capacity can become negative at low temperatures $31,32,53,54$, thus implying it cannot be proportional to a positive variance in general.

Our second result indeed shows that there are two additional contributions to the FDR due to strong-coupling (see 'Methods' section):

$$
C_{\mathcal{S}}(T)=\frac{\Delta U_{\mathcal{S}}^{2}}{T^{2}}-\frac{Q\left[\hat{\pi}_{\mathcal{S}}, \hat{E}_{\mathcal{S}}^{*}\right]}{T^{2}}+\left\langle\partial_{T} \hat{E}_{\mathcal{S}}^{*}\right\rangle,
$$

implying that $C_{\mathcal{S}}(T)$ can be less than $\Delta U_{\mathcal{S}}^{2} / T^{2}$ and even negative. We see that the first correction is due to the quantum fluctuations in energy given by the average WYD information $Q\left[\hat{\pi}_{\mathcal{S}}, \hat{E}_{\mathcal{S}}^{*}\right]$, which only vanishes in the classical limit where $\left[\hat{E}_{\mathcal{S}}^{*}(T), \hat{\pi}_{\mathcal{S}}(T)\right]=0$. The second correction is a dissipation term stemming from the temperature dependence of the internal energy operator Eq. (11). Notably this term can still be present in the classical limit where the energy operator may depend on temperature if the coupling is non-negligible. As expected both terms can be dropped in the limit of vanishing coupling and the standard FDR is recovered.

Bound on signal-to-noise ratio for temperature estimates. Let us denote the uncertainty in the temperature from a given unbiased estimation scheme by $\Delta T_{\mathcal{S}}$, with measurements performed on $\mathcal{S}$ alone. It is known $11,12,14,55$ that in the weakcoupling limit, the optimal signal-to-noise ratio for estimating $T$ from a single measurement is bounded by $C_{\mathcal{S}}(T)$ :

$$
\left(\frac{T}{\Delta T_{\mathcal{S}}}\right)^{2} \leq C_{\mathcal{S}}(T)
$$

This bound is tight for a single measurement of $T$ and implies that precise measurements of the temperature require a large heat capacity. The result follows straightforwardly from the quantum Cramér-Rao inequality and the standard FDR.

Using our modified FDR Eq. (14), we here give the strongcoupling generalisation of the bound Eq. (15). Considering estimates of $T$ rather than the inverse temperature $\beta$, a simple change of variables reveals that the QFI with respect to $T$ is related to that of $\beta, F_{\mathcal{S}}(\beta)=T^{4} F_{\mathcal{S}}(T)$. From Theorem 1 we again have $T^{4} F_{\mathcal{S}}(T) \leq K\left[\hat{\pi}_{\mathcal{S}}, \hat{E}_{\mathcal{S}}^{*}\right]$, and combining this with Eqs. (14) and (7) we obtain:

$$
\left(\frac{T}{\Delta T_{\mathcal{S}}}\right)^{2} \leq C_{\mathcal{S}}(T)-\left\langle\partial_{T} \hat{E}_{\mathcal{S}}^{*}\right\rangle .
$$

This is our third result and demonstrates that the optimal signal-to-noise ratio for estimating the temperature of $\mathcal{S}$ is bounded by both the heat capacity and the added dissipation term, which can be both positive or negative. This bound is independently tight in both the high temperature and weakcoupling limits. In these regimes the POVM saturating Eq. (16) is given by the maximum-likelihood estimator measured in the basis of the relevant symmetric logarithmic derivative ${ }^{48}$. We stress that Eq. (16) is valid in the classical limit, in which case it is always tight. We remark that the RHS of Eq. (16) can alternatively be expressed in terms of the skew information, in which case $\left(T / \Delta T_{\mathcal{S}}\right)^{2} \leq \Delta U_{\mathcal{S}}^{2} / T^{2}-Q\left[\hat{\pi}_{\mathcal{S}}, \hat{E}_{\mathcal{S}}^{*}\right] / T^{2}$. 
Application to damped harmonic oscillator. While the bound Eq. (16) is tight in the high temperature limit, for general open quantum systems the accuracy of the bound is not known. We show that the bound is very good for the example of a damped harmonic oscillator linearly coupled to $N$ harmonic oscillators in the reservoir ${ }^{34,56,57}$. Experimentally, such a model describes the behaviour of nano-mechanical resonators ${ }^{58}$ and BEC impurities $^{59}$. Here the system Hamiltonian is $\hat{H}_{\mathcal{S}}=\frac{\hat{p}^{2}}{2 M}+\frac{M \omega^{2} \hat{x}^{2}}{2}$, while the reservoir Hamiltonian is $\hat{H}_{\mathcal{R}}=\sum_{j=1}^{N}\left(\frac{\hat{p}_{j}^{2}}{2 M_{j}}+\frac{M_{j} \omega_{j}^{2} \hat{x}_{j}^{2}}{2}\right)$ and the interaction term is given by

$$
\hat{V}_{\mathcal{S} \cup \mathcal{R}}=\sum_{j=1}^{N}\left(-\lambda_{j} \hat{x} \otimes \hat{x}_{j}+\frac{\lambda_{j}^{2}}{2 M_{j} \omega_{j}^{2}} \hat{x}^{2}\right) .
$$

To allow a fully analytical solution, the reservoir frequencies are chosen equidistant, $\omega_{j}=j \Delta$ and the continuum limit is taken so that $\Delta \rightarrow 0$ (and $N \rightarrow \infty$ ). The coupling constants are chosen as the Drude-Ullersma spectrum ${ }^{34}, \lambda_{j}=\sqrt{\frac{2 \gamma M_{j} M \omega_{j}^{2} \Delta}{\pi} \frac{\omega_{D}^{2}}{\omega_{D}^{2}+\omega_{j}^{2}}}$, where $\gamma$ is the damping coefficient controlling the interaction strength and $\omega_{\mathrm{D}}$ is a large cutoff frequency.

As shown by Grabert et al. ${ }^{56}$, the resulting Hamiltonian of mean force for the oscillator can be parameterised by a temperature-dependent mass and frequency,

$$
\hat{H}_{\mathcal{S}}^{*}(T)=\frac{\hat{p}^{2}}{2 M_{T}}+\frac{M_{T} \omega_{T}^{2} \hat{x}^{2}}{2}=\hbar \omega_{T}\left(\hat{n}_{T}+\frac{1}{2}\right),
$$

where $M_{T}$ and $\omega_{T}$ are given through the expectation values of $\hat{p}^{2}$ and $\hat{x}^{2}$ in the global thermal state, see Supplementary Note 3 for detailed expressions. In its diagonal form the mean-force Hamiltonian contains a temperature-dependent number

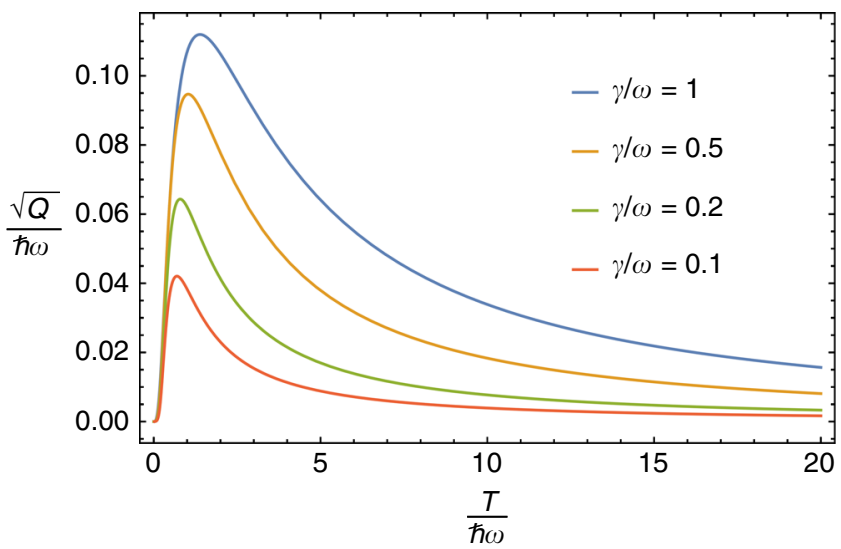

Fig. 1 Skew information for the damped oscillator. Plot of quantum energetic fluctuations $\sqrt{Q\left[\hat{\pi}_{\mathcal{S}}, \hat{E}_{\mathcal{S}}^{*}\right]} / \hbar \omega$ for the damped oscillator as a function of $T / \hbar \omega$ for different coupling strengths $\gamma$. Here $Q\left[\hat{\pi}_{\mathcal{S}}, \hat{E}_{\mathcal{S}}^{*}\right]$ is the average Wigner-Yanase-Dyson skew information for the effective energy operator $\hat{E}_{\mathcal{S}}^{*}$. These fluctuations are present when the state of the oscillator $\hat{\pi}_{\mathcal{S}}(T)$ is not diagonal in the basis of $\hat{E}_{\mathcal{S}}^{*}$ due to the non-negligible interaction between the system and reservoir. The plot shows that increasing the coupling $\gamma$ leads to an increase in the skew information. The quantum fluctuations are most pronounced at low temperatures where the thermal energies become comparable to the oscillator spacing, $T \simeq \hbar \omega$. As expected, the skew information decreases to zero in both the high temperature and weak coupling limits operator, $\quad \hat{n}_{T}=\hat{a}_{T}^{\dagger} \hat{a}_{T}$, with annihilation operator $\hat{a}_{T}=$ $\sqrt{\frac{A_{T}}{2 \hbar}}\left(\hat{x}+\frac{i}{A_{T}} \hat{p}\right)$ with $A_{T}=M_{T} \omega_{T}$.

The internal energy operator is now obtained by straightforward differentiation, see Eq. (11), and given by

$$
\hat{E}_{\mathcal{S}}^{*}(T)=\alpha_{T} \hat{H}_{\mathcal{S}}^{*}(T)-g_{T} \frac{\hat{a}_{T}^{2}+\left(\hat{a}_{T}^{\dagger}\right)^{2}}{2},
$$

where $\alpha_{T}=1-\frac{\omega_{T}^{\prime}}{\omega_{T}} T$ and $g_{T}=\hbar \omega_{T} T \frac{A_{T}^{\prime}}{A_{T}}$. Using this operator we obtain analytic expressions for $C_{\mathcal{S}}(T), F_{\mathcal{S}}(T), Q\left[\hat{\pi}_{\mathcal{S}}, \hat{E}_{\mathcal{S}}^{*}\right]$ and $\left\langle\partial_{T} \hat{E}_{\mathcal{S}}^{*}\right\rangle$ in Supplementary Note 3.

Figure 1 shows the square root of the average skew information $Q\left[\hat{\pi}_{\mathcal{S}}, \hat{E}_{\mathcal{S}}^{*}\right]$ in units of $\hbar \omega$ as a function of temperature for different coupling strengths. As expected we see that the quantum fluctuations in energy vanish in the high temperature limit, while fluctuations grow with increased coupling strengths due to increased non-commutativity between $\hat{E}_{\mathcal{S}}^{*}(T)$ and the state $\hat{\pi}_{\mathcal{S}}$ of the oscillator. Interestingly we see that $Q\left[\hat{\pi}_{\mathcal{S}}, \hat{E}_{\mathcal{S}}^{*}\right]$ decays exponentially to zero in the low temperature limit, implying that the state of the oscillator commutes with the internal energy operator in this regime. Whether this is a general feature or specific to the example here remains an open question.

Figure 2 shows the optimal signal-to-noise ratio for estimating $T$ determined by the Cramér-Rao bound Eq. (7), $\left(T / \Delta T_{\mathcal{S}}\right)_{\mathrm{opt}}^{2}=T^{2} F_{\mathcal{S}}(T)$, as a function of temperature $T$ and coupling strength $\gamma$. The bound we derived in Eq. (16) given by the heat capacity and an additional dissipation term is also plotted and shows very good agreement with the optimum estimation scheme quantified by the QFI. The bound clearly becomes tight in the high-temperature limit $(T \rightarrow \infty)$ independent of the coupling strength. Conversely the bound is also tight in the weak-coupling limit $(\gamma \rightarrow 0)$ independent of the temperature. The

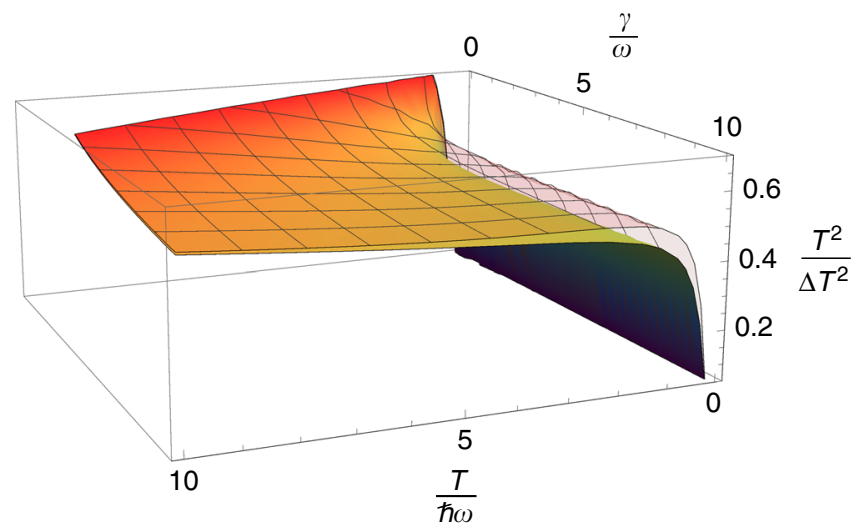

Fig. 2 Bound on temperature signal-to-noise ratio. The coloured plot shows the optimal signal-to-noise ratio $\left(T / \Delta T_{\mathcal{S}}\right)_{\text {opt }}^{2}$ of an unbiased temperature estimate for the damped oscillator, as a function of temperature $T$ and coupling strength $\gamma$. This optimal measurement is determined by the quantum Fisher information, which places an asymptotically achievable lower bound on the temperature fluctuations $\Delta T_{\mathcal{S}}$ through the Cramér-Rao inequality ${ }^{13}$. The mesh plot shows the upper bound on $\left(T / \Delta T_{\mathcal{S}}\right)_{\text {opt }}^{2}$ derived here from the generalised thermodynamic uncertainty relation Eq. (16). This uncertainty relation links the temperature fluctuations to the heat capacity of the system at arbitrary coupling strengths. It can be seen that the upper bound becomes tight in both the high temperature and weak coupling limits 
optimum and the bound both converge exponentially to zero as $T \rightarrow 0$, albeit with different rates of decay. Outside of these limits the difference between the bound and $\left(T / \Delta T_{\mathcal{S}}\right)_{\text {opt }}^{2}$ has a maximum, and at the temperature and coupling for which this maximum occurs the bound is roughly $30 \%$ greater than $\left(T / \Delta T_{\mathcal{S}}\right)_{\mathrm{opt}}^{2}$.

\section{Discussion}

In this paper we have shown how non-negligible interactions influence fluctuations in temperature at the nanoscale. Our main result Eq. (12) is a thermodynamic uncertainty relation extending the well-known complementarity relation Eq. (1) between energy and temperature to all interaction strengths. This derivation is based on a bound on the QFI for exponential states which we prove in Theorem 1. As Theorem 1 is valid for any state of fullrank, the bound will be of interest to other areas of quantum metrology. Our uncertainty relation shows that for a given finite spread in energy, unbiased estimates of the underlying temperature are limited to a greater extent due to coherences between energy states. These coherences only arise for quantum systems beyond the weak coupling assumption. We found that these additional temperature fluctuations are quantified by the average WYD skew information, thereby establishing a link between quantum and classical forms of statistical uncertainty in nanoscale thermodynamics. With coherence now understood to be an important resource in the performance of small-scale heat engines $^{60,61}$, our findings suggest that the skew information could be used to unveil further non-classical aspects of quantum thermodynamics. This complements previous results that connect skew information to both unitary phase estimation ${ }^{51}$ and quantum speed limits ${ }^{46}$.

Our second result Eq. (14) is a generalisation of the wellknown FDR to systems beyond the weak coupling regime. This further establishes a connection between the skew information and the system's heat capacity $C_{\mathcal{S}}(T)$. Proving that the heat capacity, with its strong coupling corrections, vanishes in the zero-temperature limit in accordance with the third law of thermodynamics remains an open question. The appearance of the skew information in Eq. (14) suggests that quantum coherences may play a role in ensuring its validity. Recent resource-theoretic derivations of the third law ${ }^{62,63}$ could provide a possible avenue for exploring the impact of coherences.

By applying the FDR to temperature estimation we derive our third result, Eq. (16), an upper bound on the optimal signal-tonoise ratio expressed in terms of the system's heat capacity. Notably the bound implies that when designing a probe to measure $T$, its bare Hamiltonian and interaction with the sample should be chosen so as to both maximise $C_{\mathcal{S}}(T)$ whilst minimising the additional dissipation term $\left\langle\partial_{T} \hat{E}_{\mathcal{S}}^{*}\right\rangle$. It is an interesting open question to consider the form of Hamiltonians that achieve this optimisation in the strong coupling scenario. Furthermore, one expects that improvements to low-temperature thermometry resulting from strong interactions, such as those observed in ${ }^{25}$, will be connected to the properties of the effective internal energy operator. In particular, it is clear from Eq. (16) that any improved scaling of the QFI at low temperatures must be determined by the relative scaling of $C_{\mathcal{S}}(T)$ and $\left\langle\partial_{T} \hat{E}_{\mathcal{S}}^{*}\right\rangle$, and exploring this further remains a promising direction of research. Advancements in nanotechnology now enable temperature sensing over microscopic spatial resolutions ${ }^{64,65}$, and understanding how to exploit interactions between a probe and its surroundings will be crucial to the development of these nanoscale thermometers.

The presented approach opens up opportunities for exploring the intermediate regime between the limiting cases ${ }^{66,67}$ of standard thermodynamics with negligible interactions and those

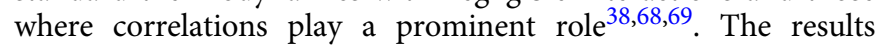
establish a connection between abstract measures of quantum information theory, such as the QFI and skew information, and a material's effective thermodynamic properties. This provides a starting point for future investigations into nanoscale thermodynamics, extending into the regime where the weak coupling assumption is not justified.

\section{Methods}

Proof of Theorem 1. Here we provide a sketch for the proof of the bound Eq. (8) on the QFI for exponential states. The full derivation can be found in Supplementary Note 1. Let us first denote the spectral decomposition of the state $\hat{\rho}_{\theta}=$ $e^{-\hat{A}_{\theta}} / Z_{\theta}$ by $\hat{\rho}_{\theta}=\sum_{n} p_{n}\left|\psi_{n}\right\rangle\left\langle\psi_{n}\right|$, where the eigenstates satisfy $\hat{A}_{\theta}\left|\psi_{n}\right\rangle=\lambda_{n}\left|\psi_{n}\right\rangle$. From this decomposition the QFI can be expressed as follows ${ }^{50}$ :

$$
F(\theta)=2 \sum_{n, m} \frac{\left|\left\langle\psi_{n}\left|\partial_{\theta} \hat{\rho}_{\theta}\right| \psi_{m}\right\rangle\right|^{2}}{p_{n}+p_{m}}
$$

To proceed we utilise the following integral expression for the derivative of an exponential operator ${ }^{70}$ :

$$
\partial_{\theta}\left[e^{-\hat{A}_{\theta}}\right]:=-\int_{0}^{1} \mathrm{~d} a e^{-(1-a) \hat{A}_{\theta}} \partial_{\theta}\left[\hat{A}_{\theta}\right] e^{-a \hat{A}_{\theta}} .
$$

Combining Eqs. (20) and (21) eventually yields

$$
F(\theta)=\Delta \hat{B}_{\theta}^{2}+\sum_{n<m}\left[\left(\frac{f^{2}\left(p_{n}, p_{m}\right)}{p_{n}+p_{m}}\right)-\left(p_{n}+p_{m}\right)\right] B_{n m}^{2},
$$

where $B_{n m}=\left|\left\langle\psi_{n}\left|\partial_{\theta} \hat{A}_{\theta}\right| \psi_{m}\right\rangle\right|, \Delta \hat{B}_{\theta}^{2}=\operatorname{Var}\left[\hat{\rho}_{\theta}, \hat{B}_{\theta}\right]$ and

$$
f\left(p_{n}, p_{m}\right):=\frac{2\left(p_{n}-p_{m}\right)}{\ln \left(p_{n} / p_{m}\right)} .
$$

Similarly, expanding in the basis $\left\{\left|\psi_{n}\right\rangle\right\}$ leads to an expression for the average skew information with respect to the operator $\hat{B}_{\theta}$ :

$$
Q\left[\hat{\rho}_{\theta}, \hat{B}_{\theta}\right]=\sum_{n<m}\left(\left(p_{n}+p_{m}\right)-f\left(p_{n}, p_{m}\right)\right) B_{n m}^{2} .
$$

Using the inequality

$$
\frac{f\left(p_{n}, p_{m}\right)}{p_{n}+p_{m}} \leq 1
$$

and comparing Eqs. (22) and (24) yields $F(\theta) \leq \Delta \hat{B}_{\theta}^{2}-Q\left[\hat{\rho}_{\theta}, \hat{B}_{\theta}\right]$. The theorem then follows from the fact that $\Delta \hat{B}_{\theta}^{2}=K\left[\hat{\rho}_{\theta}, \hat{B}_{\theta}\right]+Q\left[\hat{\rho}_{\theta}, \hat{B}_{\theta}\right]$ according to Eq. (2).

Derivation of the fluctuation-dissipation relation. Here we briefly outline the proof of the modified FDR Eq. (14). First note that according to the definition Eq. (5), the classical uncertainty in energy is given by

$$
K\left[\hat{\pi}_{\mathcal{S}}, \hat{E}_{\mathcal{S}}^{*}\right]=\int_{0}^{1} \mathrm{~d} a \operatorname{tr}\left[\hat{\pi}_{\mathcal{S}}^{1-a} \delta \hat{E}_{\mathcal{S}}^{*} \hat{\pi}_{\mathcal{S}}^{a} \delta \hat{E}_{\mathcal{S}}^{*}\right]
$$

where $\delta \hat{E}_{\mathcal{S}}^{*}:=\hat{E}_{\mathcal{S}}^{*}(T)-\left\langle\hat{E}_{\mathcal{S}}^{*}(T)\right\rangle$. By using the integral expression Eq. (21), we show in Supplementary Note 2 that this equation for $K\left[\hat{\pi}_{\mathcal{S}}, \hat{E}_{\mathcal{S}}^{*}\right]$ is equivalent to

$$
K\left[\hat{\pi}_{\mathcal{S}}, \hat{E}_{\mathcal{S}}^{*}\right]=T^{2} \operatorname{tr}\left[\hat{E}_{\mathcal{S}}^{*} \partial_{T} \hat{\pi}_{\mathcal{S}}\right] .
$$

Using the product rule for the differential of an operator, along with the equation $K\left[\hat{\pi}_{\mathcal{S}}, \hat{E}_{\mathcal{S}}^{*}\right]=\Delta U_{\mathcal{S}}^{2}-Q\left[\hat{\pi}_{\mathcal{S}}, \hat{E}_{\mathcal{S}}^{*}\right]$ given by Eq. (5), completes the derivation.

Data availability. The data that support the findings of this study are available from the corresponding author upon reasonable request.

Received: 31 January 2018 Accepted: 8 May 2018

Published online: 06 June 2018

\section{References}

1. Bohr, N. Faraday lecture. Chemistry and the quantum theory of atomic constitution. J. Chem. Soc. 1, 349-384 (1932). 
2. Mandelbrot, B. An outline of a purely phenomenological theory of statistical thermodynamics: 1. Canonical ensemble. IRE Trans. Inform. Theory 2, 190 (1956).

3. Phillies, G. D. J. The polythermal ensemble: a rigorous interpretation of temperature fluctuations in statistical mechanics. Am. J. Phys. 52, 629 (1984).

4. Velazquez, L. \& Curilef, S. Uncertainty relations of Statistical Mechanics. Phys. Rev. A. 31, 3237 (1985).

5. Lindhard, J. The Lesson of Quantum Theory. (North-Holland, Amsterdam, 1986).

6. Schlögl, F. Thermodynamic uncertainty relation. J. Phys. Chem. Solids 49, 679-683 (1988).

7. Uffink, J. B. M. \& van Lith, J. Thermodynamic uncertainty relations. Found. Phys. 29, 655-692 (1999).

8. Velazquez, L. \& Curilef, S. Uncertainty relations of Statistical Mechanics. Phys. Rev. A. 31, 2265-3239 (2009).

9. Falcioni, M., Villamaina, D., Vulpiani, A., Puglisi, A. \& Sarracino, A. Estimate of temperature and its uncertainty in small systems. Am. J. Phys. 79, 777 (2011).

10. Giazotto, F., Heikkilä, T. T., Luukanen, A., Savin, A. M. \& Pekola, J. P. Opportunities for mesoscopics in thermometry and refrigeration: physics and applications. Rev. Mod. Phys. 78, 217-274 (2006).

11. Zanardi, P., Paris, M. G. \& Campos Venuti, L. Quantum criticality as a resource for quantum estimation. Phys. Rev. A. 78, 042105 (2008).

12. Stace, T. M. Quantum limits of thermometry. Phys. Rev. A. 82, 011611(R) (2010).

13. De Pasquale, A., Rossini, D., Fazio, R. \& Giovannetti, V. Local quantum thermal susceptibility. Nat. Commun. 7, 12782 (2016).

14. Correa, L. A., Mehboudi, M., Adesso, G. \& Sanpera, A. Individual quantum probes for optimal thermometry. Phys. Rev. Lett. 114, 220405 (2015).

15. Mehboudi, M., Moreno-Cardoner, M., De Chiara, G. \& Sanpera, A. Thermometry precision in strongly correlated ultracold lattice gases. $N$. J. Phys. 17, 055020 (2015).

16. Jarzyna, M. \& Zwierz, M. Quantum interferometric measurements of temperature. Phys. Rev. A. 92, 032112 (2015).

17. Jevtic, S., Newman, D., Rudolph, T. \& Stace, T. M. Single-qubit thermometry. Phys. Rev. A. 91, 012331 (2015).

18. Carlos, L. D. \& Palacio, F. (ed.) Thermometry at the Nanoscale (The Royal Society of Chemistry, Cambridge, UK, 2016)

19. Johnson, T. H., Cosco, F., Mitchison, M. T., Jaksch, D. \& Clark, S. R. Thermometry of ultracold atoms via nonequilibrium work distributions. Phys. Rev. A. 93, 053619 (2016).

20. Campbell, S., Mehboudi, M., Chiara, G. D. \& Paternostro, M. Global and local thermometry schemes in coupled quantum systems. N. J. Phys. 19, 103003 (2017).

21. Hofer, P. P., Brask, J. B., Perarnau-Llobet, M. \& Brunner, N. Quantum thermal machine as a thermometer. Phys. Rev. Lett. 119, 090603 (2017).

22. Puglisi, A., Sarracino, A. \& Vulpiani, A. Temperature in and out of equilibrium: a review of concepts, tools and attempts. Phys. Rep. 709, 1 (2017).

23. Giovannetti, V., Lloyd, S. \& Maccone, L. Advances in quantum metrology. Nat. Photonics. 5, 222-229 (2011).

24. Marzolino, U. \& Braun, D. Precision measurements of temperature and chemical potential of quantum gases. Phys. Rev. A. 88, 063609 (2013).

25. Correa, L. A. et al. Low-temperature thermometry enhanced by strong coupling. Phys. Rev. A. 96, 062103 (2017).

26. Ludwig, M., Hammerer, K. \& Marquardt, F. Entanglement of mechanical oscillators coupled to a nonequilibrium environment. Phys. Rev. A. 82, 012333 (2010).

27. Subasi, Y., Fleming, C. H., Taylor, J. M. \& Hu, B. L. The equilibrium states of open quantum systems in the strong coupling regime. Phys. Rev. E 36, 061132 (2012).

28. Kirkwood, J. G. Statistical mechanics of fluid mixtures. J. Chem. Phys. 3, 300-313 (1935).

29. Ford, G. W., Lewis, J. T. \& O'Connell, R. F. Quantum oscillator in a blackbody radiation field. Phys. Rev. Lett. 55, 2273-2276 (1985).

30. Jarzynski, C. Nonequilibrium work theorem for a system strongly coupled to a thermal environment. J. Stat. Mech. 2004, P09005 (2004).

31. Hänggi, P., Ingold, G. L. \& Talkner, P. Finite quantum dissipation: the challenge of obtaining specific heat. N. J. Phys. 10, 115008 (2008).

32. Ingold, G., Hänggi, P. \& Talkner, P. Specific heat anomalies of open quantum systems. Phys. Rev. E 79, 061105 (2008).

33. Gelin, M. F. \& Thoss, M. Thermodynamics of a subensemble of a canonical ensemble. Phys. Rev. E 79, 051121 (2009).

34. Hilt, S., Thomas, B. \& Lutz, E. Hamiltonian of mean force for damped quantum systems. Phys. Rev. E 84, 031110 (2011).

35. Seifert, U. First and second law of thermodynamics at strong coupling. Phys. Rev. Lett. 116, 020601 (2016).

36. Jarzynski, C. Stochastic and macroscopic thermodynamics of strongly coupled systems. Phys. Rev. X 7, 011008 (2017).
37. Philbin, T. G. \& Anders, J. Thermal energies of classical and quantum damped oscillators coupled to reservoirs. J. Phys. A Math. Theor. 49, 215303 (2016)

38. Strasberg, P., Schaller, G., Lambert, N. \& Brandes, T. Nonequilibrium thermodynamics in the strong coupling and non-Markovian regime based on a reaction coordinate mapping. N. J. Phys. 18, 073007 (2016).

39. Miller, H. J. \& Anders, J. Entropy production and time asymmetry in the presence of strong interactions. Phys. Rev. E 95, 062123 (2017).

40. Strasberg, P. \& Esposito, M. Stochastic thermodynamics in the strong coupling regime: An unambiguous approach based on coarse-graining. Phys. Rev. E 95 , 062101 (2017)

41. Wigner, E. P. \& Yanase, M. M. Information contents of distributions. J. Phys Chem. 15, 1084-1259 (1963).

42. Luo, S. Heisenberg uncertainty relation for mixed states. Phys. Rev. A. 72 042110 (2005)

43. Li, X., Li, D., Huang, H., Li, X. \& Kwek, L. C. Averaged Wigner-Yanase-Dyson information as a quantum uncertainty measure. Eur. Phys. J. D 64, 147-153 (2011).

44. Frérot, I. \& Roscilde, T. Quantum variance: a measure of quantum coherence and quantum correlations for many-body systems. Phys. Rev. A 94, 075121 (2016).

45. Marvian, I. \& Spekkens, R. W. Extending Noether's theorem by quantifying the asymmetry of quantum states. Nat. Commun. 5, 3821 (2014).

46. Paiva Pires, D., Cianciaruso, M., Céleri, L., Adesso, G. \& Soares-Pinto, D. Generalized geometric quantum speed limits. Phys. Rev. X 6, 021031 (2016).

47. Luo, S. Quantum uncertainty of mixed states based on skew information. Phys. Rev. A. 73, 022324 (2006).

48. Braunstein, S. L. \& Caves, C. M. Statistical distance and the geometry of quantum states. Phys. Rev. Lett. 72, 3439 (1994).

49. Holevo, A. Probabilistic and Statistical Aspects of Quantum Theory. (NorthHolland, Amsterdam, 1982).

50. Hayashi, M. Quantum Information Theory: Mathematical Foundation. (Springer: Berlin, 2017).

51. Luo, S. Wigner-yanase skew information and uncertainty relations. Phys. Rev. Lett. 91, 180403 (2003)

52. Campisi, M., Talkner, P. \& Hänggi, P. Fluctuation theorem for arbitrary open quantum systems. Phys. Rev. Lett. 102, 210401 (2009).

53. Campisi, M., Talkner, P. \& Hänggi, P. Thermodynamics and fluctuation theorems for a strongly coupled open quantum system: an exactly solvable case. J. Phys. A Math. Theor. 42, 392002 (2009).

54. Campisi, M., Zueco, D. \& Talkner, P. Thermodynamic anomalies in open quantum systems: Strong coupling effects in the isotropic XY model. Chem. Phys. 375, 187-194 (2010).

55. Jahnke, T., Lanéry, S. \& Mahler, G. Operational approach to fluctuations of thermodynamic variables in finite quantum systems. Phys. Rev. E 83, 011109 (2011).

56. Grabert, H., Weiss, U. \& Talkner, P. Quantum theory of the damped harmonic oscillator. Z. Phys. B. 55, 87-94 (1984).

57. Pucci, L., Esposito, M. \& Peliti, L. Entropy production in quantum Brownian motion. J. Stat. Mech. 2013, P04005 (2013).

58. Connell, A. D. O. et al. Quantum ground state and single-phonon control of a mechanical resonator. Nature 464, 697 (2010).

59. Lampo, A., Lim, S. H., García-march, M. Á. \& Lewenstein, M. Bose polaron as an instance of quantum Brownian motion. Quantum 1, 30 (2017).

60. Uzdin, R., Levy, A. \& Kosloff, R. Equivalence of quantum heat machines, and quantum-thermodynamic signatures. Phys. Rev. X 5, 031044 (2015)

61. Kammerlander, P. \& Anders, J. Coherence and measurement in quantum thermodynamics. Sci. Rep. 6, 22174 (2016).

62. Masanes, L. \& Oppenheim, J. A general derivation and quantification of the third law of thermodynamics. Nat. Commun. 8, 14538 (2017).

63. Wilming, H. \& Gallego, R. Third law of thermodynamics as a single inequality. Phys. Rev. X 7, 041033 (2017).

64. Neumann, P. et al. High precision nano scale temperature sensing using single defects in diamond. Nano Lett. 13, 2738 (2013).

65. Kucsko, G. et al. Nanometer scale quantum thermometry in a living cell. Nature 500, 54 (2013)

66. González, J. O. et al. Testing the validity of the local and global GKLS master equations on an exactly solvable model. Open Syst. Inf. Dyn. 24, 1740010 (2017).

67. Hofer, P. P. et al. Markovian master equations for quantum thermal machines: local versus global approach. N. J. Phys. 19, 123037 (2017).

68. Iles-Smith, J., Lambert, N. \& Nazir, A. Environmental dynamics, correlations, and the emergence of noncanonical equilibrium states in open quantum systems. Phys. Rev. A 90, 032114 (2014).

69. Newman, D., Mintert, F. \& Nazir, A. Performance of a quantum heat engine at strong reservoir coupling. Phys. Rev. E 95, 032139 (2017).

70. Wilcox, R. M. Exponential operators and parameter differentiation in quantum physics. J. Math. Phys. 8, 962-982 (1967). 


\section{Acknowledgements}

We would like to thank Pieter Kok for insightful discussions and Simon Horsley for a critical reading of the manuscript. H.J.D.M. is supported by EPSRC through a Doctoral Training Grant. J.A. acknowledges support from EPSRC, grant EP/M009165/1, and the Royal Society. This research was supported by the COST network MP1209 "Thermodynamics in the quantum regime".

\section{Author contributions}

J.A. suggested corrections to the thermodynamic properties of strongly coupled systems and supervised the project. H.J.D.M. proved the Theorem and the generalised thermodynamic uncertainty relation. J.A. performed the example calculation. Both authors discussed the findings, drew the conclusions and wrote the paper.

\section{Additional information}

Supplementary Information accompanies this paper at https://doi.org/10.1038/s41467018-04536-7.

Competing interests: The authors declare no competing interests.

Reprints and permission information is available online at http://npg.nature.com/ reprintsandpermissions
Publisher's note: Springer Nature remains neutral with regard to jurisdictional claims in published maps and institutional affiliations.

cC (i) Open Access This article is licensed under a Creative Commons Attribution 4.0 International License, which permits use, sharing, adaptation, distribution and reproduction in any medium or format, as long as you give appropriate credit to the original author(s) and the source, provide a link to the Creative Commons license, and indicate if changes were made. The images or other third party material in this article are included in the article's Creative Commons license, unless indicated otherwise in a credit line to the material. If material is not included in the article's Creative Commons license and your intended use is not permitted by statutory regulation or exceeds the permitted use, you will need to obtain permission directly from the copyright holder. To view a copy of this license, visit http://creativecommons.org/ licenses/by/4.0/.

(C) The Author(s) 2018 